\title{
Composition of piecewise pseudo almost periodic functions and applications to abstract impulsive differential equations
}

\author{
Junwei Liu* and Chuanyi Zhang
}

\section{"Correspondence:}

junweiliuhit@gmail.com

Department of Mathematics, Harbin

Institute of Technology, Harbin,

150001, P.R. China

\begin{abstract}
In this paper, we introduce the concept of piecewise pseudo almost periodic functions on a Banach space and establish some composition theorems of piecewise pseudo almost periodic functions. We apply these composition theorems to investigate the existence of piecewise pseudo almost periodic (mild) solutions to abstract impulsive differential equations. In addition, the stability of piecewise pseudo almost periodic solutions is considered.
\end{abstract}

Keywords: piecewise pseudo almost periodic functions; composition theorem; mild solutions; impulsive differential equations

\section{Introduction}

The notion of pseudo almost periodic functions was introduced by Zhang as a natural generalization of the classical concept of almost periodic functions in $[1,2]$. Since then, such a notion has attracted many researchers' interest. The topics of these functions and their generations have been widely investigated in many publications such as [3-18] and the references therein. In particular, in [11,12], Diagana introduced the concept of Stepanov-like pseudo almost periodic functions and gave some properties including the composition theorem; the authors in [17] proposed the concept of pseudo almost periodic functions on time scales and established some results about the existence of pseudo almost periodic solutions to dynamic equations on time scales; in [10], a new concept which is called weighted pseudo-almost periodicity implements in a natural fashion the notion of pseudoalmost periodicity.

On the other hand, the study of impulsive differential equations is important [19-21] because many evolution processes, optimal control models in economics, stimulated neural networks, population models, artificial intelligence, and robotics are characterized by the fact that at certain moments of time they undergo abrupt changes of state. The existence of solutions is among the most attractive topics in the qualitative theory of impulsive differential equations [19, 21-25]. Likewise, the existence of almost periodic solutions of abstract impulsive differential equations has been considered by many authors; see, e.g., [26-28]. However, there are few papers concerned with pseudo almost periodic functions on impulsive systems.

Motivated by the above, our main propose of this paper is to introduce the concept of piecewise pseudo almost periodic functions on a Banach space and establish some com- 
position theorems of piecewise pseudo almost periodic functions. Finally, we give some results about the existence and stability of piecewise pseudo almost periodic solutions to the following abstract impulsive differential equation:

$$
\left\{\begin{array}{l}
u^{\prime}(t)=A u(t)+f(t, u(t)), \quad t \in R, t \neq t_{i}, i \in Z \\
\triangle u\left(t_{i}\right) \triangleq u\left(t_{i}^{+}\right)-u\left(t_{i}^{-}\right)=I_{i}\left(u\left(t_{i}\right)\right)
\end{array}\right.
$$

where $A$ is the infinitesimal generator of a $C_{0}$-semigroup $\{T(t): t \geq 0\}$ on a Banach space $X, f, I_{i}$, and $t_{i}$ satisfy suitable conditions that will be established later. In addition, the notations $u\left(t_{i}^{+}\right)$and $u\left(t_{i}^{-}\right)$represent the right-hand side and the left-hand side limits of $u(\cdot)$ at $t_{i}$, respectively.

\section{Preliminaries}

Throughout this paper, we denote by $X$ a Banach space; let $\mathbb{T}$ be the set consisting of all real sequences $\left\{t_{i}\right\}_{i \in Z}$ such that $\gamma=\inf _{i \in Z}\left(t_{i+1}-t_{i}\right)>0$. For $\left\{t_{i}\right\}_{i \in Z} \in \mathbb{T}$, let $P C(R, X)$ be the space formed by all bounded piecewise continuous functions $\phi: R \rightarrow X$ such that $\phi(\cdot)$ is continuous at $t$ for any $t \notin\left\{t_{i}\right\}_{i \in Z}$ and $\phi\left(t_{i}\right)=\phi\left(t_{i}^{-}\right)$for all $i \in Z$; let $\Omega$ be a subset of $X$ and $P C(R \times \Omega, X)$ be the space formed by all piecewise continuous functions $\phi: R \times \Omega \rightarrow X$ such that for any $x \in \Omega, \phi(\cdot, x) \in P C(R, X)$ and for any $t \in R, \phi(t, \cdot)$ is continuous at $x \in \Omega$.

Definition 2.1 [26] A function $\phi \in P C(R, X)$ is said to be piecewise almost periodic if the following conditions are fulfilled:

(1) $\left\{t_{i}^{j}=t_{i+j}-t_{i}\right\}, i \in Z, j=0, \pm 1, \pm 2, \ldots$, are equipotentially almost periodic; that is, for any $\epsilon>0$, there exists a relatively dense set $Q_{\epsilon}$ of $R$ such that for each $\tau \in Q_{\epsilon}$, there is an integer $q \in Z$ such that $\left|t_{i+q}-t_{i}-\tau\right|<\epsilon$ for all $i \in Z$.

(2) For any $\epsilon>0$, there exists a positive number $\delta=\delta(\epsilon)$ such that if the points $t^{\prime}$ and $t^{\prime \prime}$ belong to the same interval of continuity of $\phi$ and $\left|t^{\prime}-t^{\prime \prime}\right|<\delta$, then $\left\|\phi\left(t^{\prime}\right)-\phi\left(t^{\prime \prime}\right)\right\|<\epsilon$.

(3) For every $\epsilon>0$, there exists a relatively dense set $\Omega(\epsilon)$ in $R$ such that if $\tau \in \Omega(\epsilon)$, then

$$
\|\phi(t+\tau)-\phi(t)\|<\epsilon
$$

for all $t \in R$ satisfying the condition $\left|t-t_{i}\right|>\epsilon, i \in Z$. The number $\tau$ is called an $\epsilon$-almost period of $\phi$.

We denote by $A P_{T}(R, X)$ the space of all piecewise almost periodic functions. Obviously, the space $A P_{T}(R, X)$ endowed with the sup norm defined by $\|\phi\|_{\infty}=\sup _{t \in R}\|\phi(t)\|$ for any $\phi \in A P_{T}(R, X)$ is a Banach space.

Throughout the rest of this paper, let $\operatorname{UPC}(R, X)$ be the space of all functions $\phi \in$ $P C(R, X)$ such that $\phi$ satisfies the condition (2) in Definition 2.1.

Lemma $2.2[26]$ Let $\phi \in A P_{T}(R, X)$, then the range of $\phi, R(\phi)$, is a relatively compact subset of $X$.

Definition 2.3 $f \in P C(R \times \Omega, X)$ is said to be piecewise almost periodic in $t$ uniformly in $x \in \Omega$ if for each compact set $K \subseteq \Omega,\{f(\cdot, x): x \in K\}$ is uniformly bounded, and given 
$\epsilon>0$, there exists a relatively dense set $\Omega(\epsilon)$ such that

$$
\|f(t+\tau, x)-f(t, x)\|<\epsilon
$$

for all $x \in K, \tau \in \Omega(\epsilon)$, and $t \in R,\left|t-t_{i}\right|>\epsilon$. Denote by $A P_{T}(R \times \Omega, X)$ the set of all such functions.

Set

$$
\begin{aligned}
& \operatorname{PAP}_{T}^{0}(R, X):=\left\{\phi \in P C(R, X): \lim _{r \rightarrow \infty} \frac{1}{2 r} \int_{-r}^{r}\|\phi(t)\| d t=0\right\}, \\
& \operatorname{PAP}_{T}^{0}(R \times \Omega, X) \\
& \quad:=\left\{\Phi \in P C(R \times \Omega, X): \lim _{r \rightarrow \infty} \frac{1}{2 r} \int_{-r}^{r}\|\Phi(t, s)\| d t=0 \text { uniformly with respect to } s \in K,\right.
\end{aligned}
$$

where $K$ is an arbitrary compact subset of $\Omega\}$.

Lemma 2.4 Suppose $\phi \in P C(R, X) . \phi \in P A P_{T}^{0}(R, X)$ if and only iffor any $\epsilon>0$,

$$
\lim _{r \rightarrow \infty} \frac{m\left(M_{r, \epsilon}(\phi)\right)}{2 r}=0
$$

where $M_{r, \epsilon}(\phi)=\{t \in[-r, r]:\|\phi(t)\| \geq \epsilon\}$ and $m$ is the Lebesgue measure on $R$.

Proof Sufficiency. Since $M_{\phi}=\sup _{t \in R}\|\phi(t)\|<\infty$, by the hypothesis, for any $\epsilon>0$, there exists $r_{0}>0$ such that for $r>r_{0}$,

$$
\frac{m\left(M_{r, \epsilon}(\phi)\right)}{2 r}<\frac{\epsilon}{M_{\phi}} .
$$

Then

$$
\begin{aligned}
\frac{1}{2 r} \int_{-r}^{r}\|\phi(t)\| d t & =\frac{1}{2 r}\left[\int_{M_{r, \epsilon}(\phi)}\|\phi(t)\| d t+\int_{[-r, r] \backslash M_{r, \epsilon}(\phi)}\|\phi(t)\| d t\right] \\
& \leq \frac{m\left(M_{r, \epsilon}(\phi)\right)}{2 r} M_{\phi}+\epsilon<2 \epsilon
\end{aligned}
$$

for $r>r_{0}$. This implies that $\phi \in P A P_{T}^{0}(R, X)$.

Necessity. If it is not, there exists $\epsilon_{0}>0$ such that $\frac{m\left(M_{r, \epsilon_{0}}(\phi)\right)}{2 r} \nrightarrow 0$ as $r \rightarrow \infty$. That is, there exists $\delta>0$ such that for any $n$,

$$
\frac{m\left(M_{r_{n}, \epsilon_{0}}(\phi)\right)}{2 r_{n}} \geq \delta
$$

for some $r_{n}>n$. Then

$$
\begin{aligned}
\frac{1}{2 r_{n}} \int_{-r_{n}}^{r_{n}}\|\phi(t)\| d t & =\frac{1}{2 r_{n}}\left[\int_{M_{r_{n}, \epsilon_{0}}(\phi)}\|\phi(t)\| d t+\int_{\left[-r_{n}, r_{n}\right] \backslash M_{r_{n}, \epsilon_{0}}(\phi)}\|\phi(t)\| d t\right] \\
& \geq \frac{1}{2 r_{n}} \int_{M_{r_{n}, \epsilon_{0}}(\phi)}\|\phi(t)\| d t
\end{aligned}
$$




$$
\begin{aligned}
& \geq \frac{m\left(M_{r_{n}, \epsilon_{0}}(\phi)\right)}{2 r_{n}} \epsilon_{0} \\
& \geq \delta \epsilon_{0},
\end{aligned}
$$

which contradicts the fact that $\phi \in P A P_{T}^{0}(R, X)$. This completes the proof.

Remark 2.5 The proof of Lemma 2.4 is essentially contained in Liang et al.'s result (see Lemma 2.1 in [29]) or a more general case (see Proposition 3.1 and Corollary 3.2 in [30]). We have included it for the reader's convenience.

Lemma 2.6 $P A P_{T}^{0}(R, X)$ is a translation invariant set of $P C(R, X)$.

Proof For any $s \in R, \phi \in P A P_{T}^{0}(R, X), \epsilon>0, r>0$, we have

$$
\begin{aligned}
M_{r, \epsilon}\left(R_{s} \phi\right) & =\left\{t \in[-r, r]:\left\|R_{s} \phi(t)\right\| \geq \epsilon\right\} \\
& =\{t \in[-r, r]:\|\phi(t+s)\| \geq \epsilon\} \\
& =\{t \in[-r+s, r+s]:\|\phi(t)\| \geq \epsilon\} \subseteq\{t \in[-r-|s|, r+|s|]:\|\phi(t)\| \geq \epsilon\} .
\end{aligned}
$$

So,

$$
\frac{1}{2 r} m\left(M_{r, \epsilon}\left(R_{s} \phi\right)\right) \leq \frac{1}{2 r} m\left(M_{r+|s|, \epsilon}(\phi)\right)=\frac{2(r+|s|)}{2 r} \frac{1}{2(r+|s|)} m\left(M_{r+|s|, \epsilon}(\phi)\right) .
$$

Since $\phi \in P A P_{T}^{0}(R, X)$, then by Lemma 2.4 , we have

$$
\frac{1}{2(r+|s|)} m\left(M_{r+|s|, \epsilon}(\phi)\right) \rightarrow 0, \quad r \rightarrow \infty
$$

Furthermore, $\lim _{r \rightarrow \infty} \frac{2(r+|s|)}{2 r}=1$, so

$$
\frac{1}{2 r} m\left(M_{r, \epsilon}\left(R_{s} \phi\right)\right) \rightarrow 0, \quad r \rightarrow \infty .
$$

Again, using Lemma 2.4, we know $R_{s} \phi \in P A P_{T}^{0}(R, X)$. The proof is complete.

Definition 2.7 A function $f \in P C(R, X)$ is said to be piecewise pseudo almost periodic if it can be decomposed as $f=g+h$, where $g \in A P_{T}(R, X)$ and $h \in P A P_{T}^{0}(R, X)$. Denote by $\operatorname{PAP}_{T}(R, X)$ the set of all such functions.

$\operatorname{PAP}_{T}(R, X)$ is a Banach space with the sup norm $\|\cdot\|_{\infty}$.

Lemma 2.8 The decomposition of piecewise pseudo almost periodic functions is unique.

Proof By Definition 2.7, we only need to prove that $f \equiv 0$ when $f \in A P_{T}(R, X)$ as well as being in $\operatorname{PAP}_{T}^{0}(R, X)$. Suppose the contrary, then there exists at least one number $x_{0} \in R$ such that $f\left(x_{0}\right) \neq 0$. Without loss of generality, we may assume that $x_{0} \neq t_{i}(i \in Z)$ since we can replace $x_{0}$ by $x_{1}$ in a small neighborhood of $x_{0}$. By Lemma 76 in [20], we can choose two numbers $l>0$ and $0<\delta<\min \left\{\frac{\gamma}{2}, \min _{i \in Z}\left|x_{0}-t_{i}\right|\right\}$ such that any interval of length $l$ contains a subinterval of length $2 \delta$ whose points must all be $\frac{\left\|f\left(x_{0}\right)\right\|}{3}$-almost period and $t^{\prime}, t^{\prime \prime} \in\left(t_{i}, t_{i+1}\right)$, 
$i \in Z,\left|t^{\prime}-t^{\prime \prime}\right|<\delta$ imply that $\left\|f\left(t^{\prime}\right)-f\left(t^{\prime \prime}\right)\right\|<\frac{\left\|f\left(x_{0}\right)\right\|}{3}$. Consider now any interval of length $l$, $\left(r-\delta-x_{0}, r-\delta-x_{0}+l\right), r$ is a real number, there exists a $\frac{\left\|f\left(x_{0}\right)\right\|}{3}$-almost period $\tau$ of $f$ which belongs to this interval, thus $x_{0}+\tau \in(r-\delta, r-\delta+l)$. Assume $\left|x-x_{0}\right|<\delta$, then $x+\tau$ will range over an interval of length $2 \delta$, and

$$
\begin{aligned}
\|f(x+\tau)\| & \geq\left\|f\left(x_{0}\right)\right\|-\left\|f(x)-f\left(x_{0}\right)\right\|-\|f(x+\tau)-f(x)\| \\
& >\left\|f\left(x_{0}\right)\right\|-\frac{\left\|f\left(x_{0}\right)\right\|}{3}-\frac{\left\|f\left(x_{0}\right)\right\|}{3}=\frac{\left\|f\left(x_{0}\right)\right\|}{3},
\end{aligned}
$$

which shows that any interval of length $l$ contains a subinterval of length $2 \delta$ at all points satisfying $\|f(x)\|>\frac{\left\|f\left(x_{0}\right)\right\|}{3}$, so

$$
\frac{1}{2 n l} \int_{-n l}^{n l}\|f(x)\| d x \geq \frac{1}{2 n l} \sum_{k=-(n-1)}^{n} \int_{(k-1) l}^{k l}\|f(x)\| d x>\frac{1}{2 n l} \cdot 2 n \cdot 2 \delta \cdot \frac{\left\|f\left(x_{0}\right)\right\|}{3}=\frac{2 \delta\left\|f\left(x_{0}\right)\right\|}{3 l},
$$

which contradicts the fact that $f \in P A P_{T}^{0}(R, X)$. The proof is complete.

Definition 2.9 Let $P A P_{T}(R \times \Omega, X)$ consist of all functions $F \in P C(R \times \Omega, X)$ such that $F=G+H$, where $G \in A P_{T}(R \times \Omega, X)$ and $H \in P A P_{T}^{0}(R \times \Omega, X)$.

\section{Composition theorems}

Theorem 3.1 Suppose $f \in P A P_{T}(R \times \Omega, X)$. Assume that the following conditions hold:

(i) $\{f(t, x): t \in R, x \in K\}$ is bounded for every bounded subset $K \subseteq \Omega$.

(ii) $f(t, \cdot)$ is uniformly continuous in each bounded subset of $\Omega$ uniformly in $t \in R$.

If $\phi \in P A P_{T}(R, X)$ such that $R(\phi) \subset \Omega$, then $f(\cdot, \phi(\cdot)) \in P_{P A} P_{T}(R, X)$.

Proof Since $f \in P A P_{T}(R \times \Omega, X)$ and $\phi \in P A P_{T}(R, X)$, by Definitions 2.7 and 2.9, we have $f=g+h$ and $\phi=\phi_{1}+\phi_{2}$ with $g \in A P_{T}(R \times \Omega, X), h \in P_{A} P_{T}^{0}(R \times \Omega, X), \phi_{1} \in A P_{T}(R, X)$, $\phi_{2} \in P A P_{T}^{0}(R, X)$. So, the function $f(\cdot, \phi(\cdot))$ can be decomposed as

$$
\begin{aligned}
f(\cdot, \phi(\cdot)) & =g\left(\cdot, \phi_{1}(\cdot)\right)+f(\cdot, \phi(\cdot))-g\left(\cdot, \phi_{1}(\cdot)\right) \\
& =g\left(\cdot, \phi_{1}(\cdot)\right)+f(\cdot, \phi(\cdot))-f\left(\cdot, \phi_{1}(\cdot)\right)+h\left(\cdot, \phi_{1}(\cdot)\right) .
\end{aligned}
$$

By Lemma 2.2, $R\left(\phi_{1}\right)$ is relatively compact in $X, g(t, \cdot)$ is uniformly continuous in $R\left(\phi_{1}\right)$ uniformly in $t \in R$. By a proof similar to Theorem 3.1 in [27], $g\left(\cdot, \phi_{1}(\cdot)\right) \in A P_{T}(R, X)$. To show that $f(\cdot, \phi(\cdot)) \in P A P_{T}(R, X)$, we need to show that $f(\cdot, \phi(\cdot))-f\left(\cdot, \phi_{1}(\cdot)\right)+h\left(\cdot, \phi_{1}(\cdot)\right) \in$ $\operatorname{PAP}_{T}^{0}(R, X)$.

First, we show that $f(\cdot, \phi(\cdot))-f\left(\cdot, \phi_{1}(\cdot)\right) \in P A P_{T}^{0}(R, X)$. Let $K$ be a bounded subset of $\Omega$ such that $R(\phi) \subseteq K, R\left(\phi_{1}\right) \subseteq K$. By (ii), $f(t, \cdot)$ is uniformly continuous in $R\left(\phi_{1}\right)$ uniformly in $t \in R$, given $\epsilon>0$, there exists $\delta>0$ such that $y_{1}, y_{2} \in K$ and $\left\|y_{1}-y_{2}\right\|<\delta$ implies that

$$
\left\|f\left(t, y_{1}\right)-f\left(t, y_{2}\right)\right\|<\epsilon, \quad t \in R .
$$

Thus, for each $t \in R,\left\|\phi_{2}(t)\right\|<\delta$ implies that for all $t \in R$,

$$
\left\|f(t, \phi(t))-f\left(t, \phi_{1}(t)\right)\right\|<\epsilon,
$$


where $\phi_{2}(t)=\phi(t)-\phi_{1}(t)$. For $r>0$, let $M_{r, \delta}\left(\phi_{2}\right)=\left\{t \in[-r, r]:\left\|\phi_{2}(t)\right\| \geq \delta\right\}$, so we get

$$
M_{r, \epsilon}\left(f, \phi, \phi_{1}\right) \triangleq M_{r, \epsilon}\left(f(\cdot, \phi(\cdot))-f\left(\cdot, \phi_{1}(\cdot)\right)\right) \subseteq M_{r, \delta}\left(\phi_{2}\right) .
$$

Since $\phi_{2} \in P A P_{T}^{0}(R, X)$, by Lemma 2.4, we have

$$
\frac{m\left(M_{r, \delta}\left(\phi_{2}\right)\right)}{2 r} \rightarrow 0 \quad \text { as } r \rightarrow \infty
$$

hence

$$
\frac{m\left(M_{r, \epsilon}\left(f, \phi, \phi_{1}\right)\right)}{2 r} \rightarrow 0 \quad \text { as } r \rightarrow \infty
$$

this implies $f(\cdot, \phi(\cdot))-f\left(\cdot, \phi_{1}(\cdot)\right) \in P A P_{T}^{0}(R, X)$ by Lemma 2.4 .

It remains to show $h\left(\cdot, \phi_{1}(\cdot)\right) \in P A P_{T}^{0}(R, X)$. Note that $f=g+h$ and $g(t, \cdot)$ is uniformly continuous in $R\left(\phi_{1}\right)$ uniformly in $t \in R$. By the hypothesis (ii), $f(t, \cdot)$ is uniformly continuous in $R\left(\phi_{1}\right)$ uniformly in $t \in R$, so is $h$. Since $R\left(\phi_{1}\right)$ is relatively compact in $\mathrm{X}$, for $\epsilon>0$, one can find a finite number $n$ of open balls $O_{k}$ with center $z_{k} \in R\left(x_{1}\right), k=1,2, \ldots, n$ and radium $\delta\left(z_{k}, \frac{\epsilon}{2}\right)$ such that $R\left(\phi_{1}\right) \subseteq \bigcup_{k=1}^{n} O_{k}$ and

$$
\left\|h(t, z)-h\left(t, z_{k}\right)\right\|<\frac{\epsilon}{2}, \quad z \in O_{k}, t \in R .
$$

The set

$$
B_{k}=\left\{t \in R: \phi_{1}(t) \in O_{k}\right\}
$$

is open and $R=\bigcup_{k=1}^{n} B_{k}$, let $E_{k}=B_{k} \backslash \bigcup_{j=1}^{k-1} B_{j}$, then $E_{i} \bigcap E_{j}=\emptyset$ when $i \neq j, 1 \leq i, j \leq n$.

Since each $h\left(\cdot, z_{k}\right) \in P A P_{T}^{0}(R, X)$, there is a number $r_{0}$ such that

$$
\sum_{k=1}^{n} \frac{1}{2 r} \int_{-r}^{r}\left\|h\left(t, z_{k}\right)\right\| d t<\frac{\epsilon}{2}, \quad r>r_{0}
$$

Then

$$
\begin{aligned}
\frac{1}{2 r} \int_{-r}^{r}\left\|h\left(t, \phi_{1}(t)\right)\right\| d t= & \frac{1}{2 r} \sum_{k=1}^{n} \int_{E_{n} \cap[-r, r]}\left\|h\left(t, \phi_{1}(t)\right)\right\| d t \\
\leq & \frac{1}{2 r} \sum_{k=1}^{n}\left[\int_{E_{n} \cap[-r, r]}\left\|h\left(t, \phi_{1}(t)\right)-h\left(t, z_{k}\right)\right\| d t\right. \\
& \left.+\int_{E_{n} \cap[-r, r]}\left\|h\left(t, z_{k}\right)\right\| d t\right] \\
\leq & \frac{\epsilon}{2}+\sum_{k=1}^{n} \frac{1}{2 r} \int_{-r}^{r}\left\|h\left(t, z_{k}\right)\right\| d t \\
& <\frac{\epsilon}{2}+\frac{\epsilon}{2}=\epsilon .
\end{aligned}
$$

This implies that $h\left(\cdot, \phi_{1}(\cdot)\right) \in P A P_{T}^{0}(R, X)$. Thus, $f(\cdot, \phi(\cdot)) \in P A P_{T}(R, X)$. This completes the proof. 
Remark 3.2 A result similar to Theorem 3.1 was obtained by Agarwal et al. for weighted pseudo-almost periodic functions (see Theorem 3.2 in [30]).

Since the uniform continuity is weaker than the Lipschitz continuity, the next corollary is a straightforward consequence of the previous theorem.

Corollary 3.3 Let $f \in P A P_{T}(R \times \Omega, X), \phi \in \operatorname{PAP}_{T}(R, X)$ and $R(\phi) \subset \Omega$. Assume further that there exists a number $L>0$ satisfying

$$
\|f(t, x)-f(t, y)\| \leq L\|x-y\|, \quad \forall t \in R, x, y \in \Omega,
$$

then the function $t \mapsto f(t, \phi(t))$ belongs to $P A P_{T}(R, X)$.

Theorem 3.4 Assume the sequence of vector-valued functions $\left\{I_{i}\right\}_{i \in Z}$ is pseudo almost periodic, i.e., for any $x \in \Omega,\left\{I_{i}(x), i \in Z\right\}$ is a pseudo almost periodic sequence. Suppose $\left\{I_{i}(x): i \in Z, x \in K\right\}$ is bounded for every bounded subset $K \subseteq \Omega, I_{i}(x)$ is uniformly continuous in $x \in \Omega$ uniformly in $i \in Z$. If $\phi \in P A P_{T}(R, X) \cap U P C(R, X)$ such that $R(\phi) \subset \Omega$, then $I_{i}\left(\phi\left(t_{i}\right)\right)$ is pseudo almost periodic.

Proof Fix $\phi \in P A P_{T}(R, X) \cap U P C(R, X)$, first we show $\phi\left(t_{i}\right)$ is pseudo almost periodic. By Definition 2.7, we have $\phi=\phi_{1}+\phi_{2}$, where $\phi_{1} \in A P_{T}(R, X), \phi_{2} \in P A P_{T}^{0}(R, X)$. It follows from Lemma 37 in [20] that the sequence $\phi_{1}\left(t_{i}\right)$ is almost periodic. To show $\phi\left(t_{i}\right)$ is pseudo almost periodic, we need to show that $\phi_{2}\left(t_{i}\right) \in P A P_{0}(Z, X)$. By the hypothesis, $\phi, \phi_{1} \in U P C(R, X)$, so is $\phi_{2}$. Let $0<\epsilon<1$, there exists $0<\delta<\min \{1, \gamma\}$ such that for $t \in\left(t_{i}-\delta, t_{i}\right), i \in Z$, we have

$$
\left\|\phi_{2}(t)\right\| \geq(1-\epsilon)\left\|\phi_{2}\left(t_{i}\right)\right\|, \quad i \in Z
$$

Since $\left\{t_{i}^{j}\right\}, i \in Z, j=0, \pm 1, \pm 2, \ldots$ are equipotentially almost periodic, $\left\{t_{i}^{1}\right\}$ is an almost periodic sequence. Here we assume a bound of $\left\{t_{i}^{1}\right\}$ is $M_{t}$ and $\left|t_{i}\right| \geq\left|t_{-i}\right|$; therefore,

$$
\begin{aligned}
\frac{1}{2 t_{i}} \int_{-t_{i}}^{t_{i}}\left\|\phi_{2}(t)\right\| d t & \geq \frac{1}{2 t_{i}} \sum_{j=-i+1}^{i} \int_{t_{j-1}}^{t_{j}}\left\|\phi_{2}(t)\right\| d t \\
& \geq \frac{1}{2 t_{i}} \sum_{j=-i+1}^{i} \int_{t_{j}-\delta}^{t_{j}}\left\|\phi_{2}(t)\right\| d t \\
& \geq \frac{1}{2 t_{i}} \sum_{j=-i+1}^{i} \delta(1-\epsilon)\left\|\phi_{2}\left(t_{j}\right)\right\| \\
& \geq \frac{\delta(1-\epsilon)}{M_{t}} \frac{1}{2 i} \sum_{j=-i+1}^{i}\left\|\phi_{2}\left(t_{j}\right)\right\| .
\end{aligned}
$$

Since $\phi_{2} \in P A P_{T}^{0}(R, X)$, it follows from the inequality above that $\phi_{2}\left(t_{i}\right) \in P A P_{0}(Z, X)$. Hence, $\phi\left(t_{i}\right)$ is pseudo almost periodic. 
Now, we show $I_{i}\left(\phi\left(t_{i}\right)\right)$ is pseudo almost periodic. Let

$$
\begin{array}{ll}
I(t, x)=I_{n}(x)+(t-n)\left[I_{n+1}(x)-I_{n}(x)\right], & n \leq t<n+1, n \in Z, \\
\Phi(t)=\phi\left(t_{n}\right)+(t-n)\left[\phi\left(t_{n+1}\right)-\phi\left(t_{n}\right)\right], & n \leq t<n+1, n \in Z .
\end{array}
$$

Since $I_{n}, \phi\left(t_{n}\right)$ are two pseudo almost periodic sequences, by Lemma 1.7.12 in [31], we know that $I \in P A P(R \times \Omega, X), \Phi \in P A P(R, X)$. For every $t \in R$, there exists a number $n \in Z$ such that $|t-n| \leq 1$,

$$
\begin{aligned}
\|I(t, x)\| & \leq\left\|I_{n}(x)\right\|+|t-n|\left[\left\|I_{n+1}(x)\right\|+\left\|I_{n}(x)\right\|\right] \\
& \leq 2\left\|I_{n}(x)\right\|+\left\|I_{n+1}(x)\right\| .
\end{aligned}
$$

Since $\left\{I_{n}(x): n \in Z, x \in K\right\}$ is bounded for every bounded set $K \subseteq \Omega,\{I(t, x): t \in R, x \in K\}$ is bounded for every bounded set $K \subseteq \Omega$. For every $x_{1}, x_{2} \in \Omega$, we have

$$
\begin{aligned}
& \left\|I\left(t, x_{1}\right)-I\left(t, x_{2}\right)\right\| \\
& \quad \leq\left\|I_{n}\left(x_{1}\right)-I_{n}\left(x_{2}\right)\right\|+|t-n|\left[\left\|I_{n+1}\left(x_{1}\right)-I_{n+1}\left(x_{2}\right)\right\|+\left\|I_{n}\left(x_{1}\right)-I_{n}\left(x_{2}\right)\right\|\right] \\
& \quad \leq 2\left\|I_{n}\left(x_{1}\right)-I_{n}\left(x_{2}\right)\right\|+\left\|I_{n+1}\left(x_{1}\right)-I_{n+1}\left(x_{2}\right)\right\| .
\end{aligned}
$$

Noting that $I_{i}(x)$ is uniformly continuous in $x \in \Omega$ uniformly in $i \in Z$, we then get that $I(t, x)$ is uniformly continuous in $x \in \Omega$ uniformly in $t \in R$. Then by Theorem 2.1 in [15], $I(\cdot, \Phi(\cdot)) \in P A P(R, X)$. Again, using Lemma 1.7.12 in [31], we have that $I(i, \Phi(i))$ is a pseudo almost periodic sequence, that is, $I_{i}\left(\phi\left(t_{i}\right)\right)$ is pseudo almost periodic. This completes the proof.

Corollary 3.5 Assume the sequence of vector-valued functions $\left\{I_{i}\right\}_{i \in Z}$ is pseudo almost periodic, if there is a number $L>0$ such that

$$
\left\|I_{i}(x)-I_{i}(y)\right\| \leq L\|x-y\|
$$

for all $x, y \in \Omega, i \in Z$, if $\phi \in P A P_{T}(R, X) \cap U P C(R, X)$ such that $R(\phi) \subset \Omega$, then $I_{i}\left(\phi\left(t_{i}\right)\right)$ is pseudo almost periodic.

\section{Piecewise pseudo almost periodic solutions}

In this section, we investigate the existence and stability of a piecewise pseudo almost periodic solution to Eq. (1.1). Before starting our main results in this section, we recall the definition of a mild solution to Eq. (1.1).

Definition 4.1 A function $u: R \rightarrow X$ is called a mild solution of Eq. (1.1) if for any $t \in R$, $t>\sigma, \sigma \neq t_{i}, i \in Z$,

$$
u(t)=T(t-\sigma) u(\sigma)+\int_{\sigma}^{t} T(t-s) f(s, u(s)) d s+\sum_{\sigma<t_{i}<t} T\left(t-t_{i}\right) I_{i}\left(u\left(t_{i}\right)\right) .
$$


In fact, using the semigroup theory, we know

$$
u(t)=T(t-\sigma) u(\sigma)+\int_{\sigma}^{t} T(t-s) f(s, u(s)) d s, \quad t>\sigma,
$$

is a mild solution to

$$
u^{\prime}(t)=A u(t)+f(t, u(t))
$$

For any $\sigma \in R$, we can find $i \in Z, t_{i-1}<\sigma \leq t_{i}$, for $t \in\left(\sigma, t_{i}\right]$,

$$
u(t)=T(t-\sigma) u(\sigma)+\int_{\sigma}^{t} T(t-s) f(s, u(s)) d s
$$

by using $u\left(t_{i}^{+}\right)-u\left(t_{i}^{-}\right)=I_{i}\left(u\left(t_{i}\right)\right)$, we have

$$
u\left(t_{i}^{+}\right)=T\left(t_{i}-\sigma\right) u(\sigma)+\int_{\sigma}^{t_{i}} T\left(t_{i}-s\right) f(s, u(s)) d s+I_{i}\left(u\left(t_{i}\right)\right),
$$

then we have

$$
\begin{aligned}
u(t)= & T\left(t-t_{i}\right) u\left(t_{i}^{+}\right)+\int_{t_{i}}^{t} T(t-s) f(s, u(s)) d s \\
= & T\left(t-t_{i}\right)\left[T\left(t_{i}-\sigma\right) u(\sigma)+\int_{\sigma}^{t_{i}} T\left(t_{i}-s\right) f(s, u(s)) d s+I_{i}\left(u\left(t_{i}\right)\right)\right] \\
& +\int_{t_{i}}^{t} T(t-s) f(s, u(s)) d s \\
= & T(t-\sigma) u(\sigma)+\int_{\sigma}^{t_{i}} T(t-s) f(s, u(s)) d s+T\left(t-t_{i}\right) I_{i}\left(u\left(t_{i}\right)\right) \\
& +\int_{t_{i}}^{t} T(t-s) f(s, u(s)) d s \\
= & T(t-\sigma) u(\sigma)+\int_{\sigma}^{t} T(t-s) f(s, u(s)) d s+T\left(t-t_{i}\right) I_{i}\left(u\left(t_{i}\right)\right) .
\end{aligned}
$$

Reiterating this procedure, we get

$$
u(t)=T(t-\sigma) u(\sigma)+\int_{\sigma}^{t} T(t-s) f(s, u(s)) d s+\sum_{\sigma<t_{i}<t} T\left(t-t_{i}\right) I_{i}\left(u\left(t_{i}\right)\right)
$$

First, we study the existence of a piecewise pseudo almost periodic mild solution of Eq. (1.1) when the perturbations $f, I_{i}(i \in Z)$ are not Lipschitz continuous. We need a criterion of the relatively compact set in $P C(R, X)$. We list the following result about the relatively compact set; one may refer to [32-34] for more details.

Let $h: R \rightarrow R$ be a continuous function such that $h(t) \geq 1$ for all $t \in R$ and $h(t) \rightarrow \infty$ as $|t| \rightarrow \infty$. We consider the space

$$
(P C)_{h}^{0}(R, X)=\left\{\phi \in P C(R, X): \lim _{|t| \rightarrow \infty} \frac{\|\phi(t)\|}{h(t)}=0\right\} .
$$

Endowed with the norm $\|\phi\|_{h}=\sup _{t \in R} \frac{\|\phi(t)\|}{h(t)}$, it is a Banach space. 
Lemma 4.2 A set $B \subseteq(P C)_{h}^{0}(R, X)$ is a relatively compact set if and only if

(1) $\lim _{|t| \rightarrow \infty} \frac{\|\phi(t)\|}{h(t)}=0$ uniformly for $\phi \in B$.

(2) $B(t)=\{\phi(t): \phi \in B\}$ is relatively compact in $X$ for every $t \in R$.

(3) The set $B$ is equicontinuous on each interval $\left(t_{i}, t_{i+1}\right)(i \in Z)$.

Proof Let $(P C)^{0}(R, X)=\left\{\phi \in P C(R, X): \lim _{|t| \rightarrow \infty}\|\phi(t)\|=0\right\}$. By an analogous argument in $[33,34],(P C)^{0}(R, X)$ is isometrically isomorphic with the space $(P C)_{h}^{0}(R, X)$. In order to prove Lemma 4.2, we only need to show that $B^{\prime} \subseteq(P C)^{0}(R, X)$ is a relatively compact set if and only if

(11) $\lim _{|t| \rightarrow \infty}\|f(t)\|=0$ uniformly for $f \in B^{\prime}$.

(22) $B^{\prime}(t)=\left\{f(t): f \in B^{\prime}\right\}$ is relatively compact in $X$ for every $t \in R$.

(33) The set $B^{\prime}$ is equicontinuous on each interval $\left(t_{i}, t_{i+1}\right)(i \in Z)$.

Sufficiency. By (11), for any $\epsilon>0$, there exists $\delta_{1}>0$ such that

$$
\|f(t)\|<\epsilon, \quad|t|>\delta_{1}, f \in B^{\prime}
$$

By (33), for the above $\epsilon$, there exists $\delta: 0<\delta<\min \left\{\delta_{1}, \gamma\right\}$ such that $t^{\prime}, t^{\prime \prime} \in\left(t_{i}, t_{i+1}\right), i \in Z$, $\left|t^{\prime}-t^{\prime \prime}\right|<\delta$,

$$
\left\|f\left(t^{\prime}\right)-f\left(t^{\prime \prime}\right)\right\|<\epsilon, \quad f \in B^{\prime} .
$$

For the interval $\left[-\delta_{1}, \delta_{1}\right]$, there exists a set $S=\left\{s_{1}, s_{2}, \ldots, s_{q}\right\} \subset\left[-\delta_{1}, \delta_{1}\right], s_{j} \neq t_{i}, j=1,2, \ldots, q$ such that $\left|t-s_{j}\right|<\delta$ and

$$
\left\|f(t)-f\left(s_{j}\right)\right\|<\epsilon, \quad j=1,2, \ldots, q, f \in B^{\prime} .
$$

For any sequence $\left\{f_{k}: k \geq 1\right\} \subseteq B^{\prime}$, by (22), we can extract a subsequence that converges at each point $t \in R$. Since $S$ is finite, then for the above $\epsilon>0$, there exists $n_{0} \in N$,

$$
\left\|f_{m}(t)-f_{n}(t)\right\|<\epsilon, \quad m, n \geq n_{0}, t \in S
$$

So, for $t \in\left[-\delta_{1}, \delta_{1}\right]$, by (4.2) and (4.3),

$$
\left\|f_{m}(t)-f_{n}(t)\right\| \leq\left\|f_{m}(t)-f_{m}\left(s_{j}\right)\right\|+\left\|f_{m}\left(s_{j}\right)-f_{n}\left(s_{j}\right)\right\|+\left\|f_{n}\left(s_{j}\right)-f_{n}(t)\right\|<3 \epsilon .
$$

For $|t|>\delta_{1}$, by $(4.1)$,

$$
\left\|f_{m}(t)-f_{n}(t)\right\|<2 \epsilon
$$

Thus, $\left\{f_{k}: k \geq 1\right\}$ is uniformly convergent on $R, B^{\prime} \subseteq(P C)^{0}(R, X)$ is a relatively compact set.

Necessity. Since $B^{\prime} \subseteq(P C)^{0}(R, X)$ is relatively compact, for any $\epsilon>0$, there exist a finite number of functions $f_{1}, f_{2}, \ldots, f_{m}$ of $B^{\prime}$ such that

$$
\left\|f-f_{j}\right\|<\epsilon, \quad j=1,2, \ldots, m, f \in B^{\prime} .
$$


This finite set of functions $f_{1}, f_{2}, \ldots, f_{m}$ is equicontinuous; that is, for the above $\epsilon$, there exists a number $\delta_{2}>0$ such that $t^{\prime}, t^{\prime \prime} \in\left(t_{i}, t_{i+1}\right), i \in Z,\left|t^{\prime}-t^{\prime \prime}\right|<\delta_{2}$, we have $\left\|f\left(t^{\prime}\right)-f\left(t^{\prime \prime}\right)\right\|<$ $\epsilon$, using (4.4), for any $f \in B^{\prime}$,

$$
\left\|f\left(t^{\prime}\right)-f\left(t^{\prime \prime}\right)\right\| \leq\left\|f\left(t^{\prime}\right)-f_{j}\left(t^{\prime}\right)\right\|+\left\|f_{j}\left(t^{\prime}\right)-f_{j}\left(t^{\prime \prime}\right)\right\|+\left\|f_{j}\left(t^{\prime \prime}\right)-f\left(t^{\prime \prime}\right)\right\|<3 \epsilon
$$

which shows (33). Since $f_{j} \in B^{\prime}$, then for the above $\epsilon$, there exist numbers $\zeta_{j}>0$ such that

$$
\left\|f_{j}(t)\right\|<\epsilon, \quad|t|>\zeta_{j}, j=1,2, \ldots, m \text {. }
$$

Let $\delta_{3}=\max \left\{\zeta_{1}, \zeta_{2}, \ldots, \zeta_{m}\right\}$, by (4.4) and (4.5), for any $f \in B^{\prime}$,

$$
\|f(t)\| \leq\left\|f(t)-f_{j}(t)\right\|+\left\|f_{j}(t)\right\|<2 \epsilon, \quad|t|>\delta_{3}
$$

which shows (11). Since $B^{\prime}$ is relatively compact, then for any sequence $\left\{f_{k}: k \geq 1\right\} \subseteq B^{\prime}$, there exists a subsequence that converges uniformly on $R$. Fix $t \in R$, from the sequence $\left\{f_{k}(t): k \geq 1\right\} \subseteq X$, there exists a convergent subsequence. Therefore, for fixed $t \in R$, the set $\left\{f(t): f \in B^{\prime}\right\}$ is relatively compact, which shows (22). The proof is complete.

Remark 4.3 In the $C(R, X)$-setting, a result similar to Lemma 4.2 was proved in Henriquez and Lizama [33] (see also [34]).

The first existence result is based upon the Schauder fixed point theorem.

Theorem 4.4 Suppose Eq. (1.1) satisfies the following conditions:

(A1) $A$ is the infinitesimal generator of an exponentially stable $C_{0}$-semigroup $\{T(t): t \geq 0\}$; that is, there exist numbers $\delta, M>0$ such that $\|T(t)\| \leq M e^{-\delta t}, t \geq 0$. Moreover, $T(t)$ is compact for $t>0$.

(A2) $f \in P A P_{T}(R \times \Omega, X)$, and $f(t, \cdot)$ is uniformly continuous in each bounded subset of $\Omega$ uniformly in $t \in R ; I_{i}$ is a pseudo almost periodic sequence, $I_{i}(x)$ is uniformly continuous in $x \in \Omega$ uniformly in $i \in Z$.

(A3) For any $L>0, C_{1 L}=\sup _{t \in R,\|x\| \leq L}\|f(t, x)\|<\infty, C_{2 L}=\sup _{i \in Z,\|x\| \leq L}\left\|I_{i}(x)\right\|<\infty$. Moreover, there exists a number $L_{0}>0$ such that $\frac{M}{\delta} C_{1 L_{0}}+\frac{M}{1-e^{-\delta \gamma}} C_{2 L_{0}} \leq L_{0}$.

Then Eq. (1.1) has a piecewise pseudo almost periodic solution.

Proof Let $D=\left\{\phi \in P A P_{T}(R, X) \cap U P C(R, X):\|\phi\| \leq L_{0}\right\}$. Define an operator $\Gamma$ on $D$ by

$$
\Gamma \phi(t)=\int_{-\infty}^{t} T(t-s) f(s, \phi(s)) d s+\sum_{t_{i}<t} T\left(t-t_{i}\right) I_{i}\left(\phi\left(t_{i}\right)\right)
$$

We next show that $\Gamma$ has a fixed point in $D$. We divide the proof into several steps.

Step 1 . For every $\phi \in D, \Gamma \phi \in P A P_{T}(R, X)$.

Fix $\phi \in D$, by (A2) and Theorem 3.1, we have $f(\cdot, \phi(\cdot)) \in P A P_{T}(R, X)$, then we have by Definition 2.7 that $f(\cdot, \phi(\cdot))=\phi_{1}(\cdot)+\phi_{2}(\cdot)$, where $\phi_{1} \in A P_{T}(R, X), \phi_{2} \in P A P_{T}^{0}(R, X)$, so

$$
\int_{-\infty}^{t} T(t-s) f(s, \phi(s)) d s=\int_{-\infty}^{t} T(t-s) \phi_{1}(s) d s+\int_{-\infty}^{t} T(t-s) \phi_{2}(s) d s \triangleq I_{1}(t)+I_{2}(t) .
$$


Meanwhile, given $\epsilon>0$, there exists a relatively dense set $\Omega_{1}$ such that for $\tau \in \Omega_{1}, t \in R$, $\left|t-t_{i}\right|>\epsilon$,

$$
\left\|\phi_{1}(t+\tau)-\phi_{1}(t)\right\|<\epsilon
$$

Thus, by (A1),

$$
\begin{aligned}
\left\|I_{1}(t+\tau)-I_{1}(t)\right\| & =\left\|\int_{-\infty}^{t+\tau} T(t+\tau-s) \phi_{1}(s) d s-\int_{-\infty}^{t} T(t-s) \phi_{1}(s) d s\right\| \\
& =\left\|\int_{-\infty}^{t} T(t-s)\left[\phi_{1}(s+\tau)-\phi_{1}(s)\right] d s\right\| \\
& \leq \int_{-\infty}^{t} M e^{-\delta(t-s)}\left\|\phi_{1}(s+\tau)-\phi_{1}(s)\right\| d s \\
& \leq \frac{M}{\delta} \epsilon,
\end{aligned}
$$

this implies that $I_{1} \in A P_{T}(R, X)$. Since $P A P_{T}^{0}(R, X)$ is translation invariant, then for $\phi_{2} \in$ $P A P_{T}^{0}(R, X)$, one can find $r_{0}>0$ such that

$$
\frac{1}{2 r} \int_{-r}^{r}\left\|\phi_{2}(t-s)\right\| d t<\epsilon, \quad r>r_{0}, s \in R .
$$

Then by (A1), one obtains that

$$
\begin{aligned}
\frac{1}{2 r} \int_{-r}^{r}\left\|I_{2}(t)\right\| d t & =\frac{1}{2 r} \int_{-r}^{r}\left\|\int_{-\infty}^{t} T(t-s) \phi_{2}(s) d s\right\| d t \\
& \leq \frac{1}{2 r} \int_{-r}^{r} d t \int_{-\infty}^{t} M e^{-\delta(t-s)}\left\|\phi_{2}(s)\right\| d s \\
& =\frac{1}{2 r} \int_{-r}^{r} d t \int_{0}^{\infty} M e^{-\delta s}\left\|\phi_{2}(t-s)\right\| d s \\
& =\int_{0}^{\infty} M e^{-\delta s} d s \frac{1}{2 r} \int_{-r}^{r}\left\|\phi_{2}(t-s)\right\| d t .
\end{aligned}
$$

By using the Lebesgue dominated convergence theorem and (4.6), we have $I_{2} \in P A P_{T}^{0}(R$, $X)$. Thus, $\int_{-\infty} T(\cdot-s) f(s, \phi(s)) d s \in \operatorname{PAP}_{T}(R, X)$.

Moreover, by (A3) and Theorem 3.4, $I_{i}\left(\phi\left(t_{i}\right)\right)$ is a pseudo almost periodic sequence, then $I_{i}\left(\phi\left(t_{i}\right)\right)=\beta_{i}+\gamma_{i}$ with $\beta_{i}$ is an almost periodic sequence and $\gamma_{i} \in P A P_{0}(Z, X)$, so

$$
\sum_{t_{i}<t} T\left(t-t_{i}\right) I_{i}\left(\phi\left(t_{i}\right)\right)=\sum_{t_{i}<t} T\left(t-t_{i}\right) \beta_{i}+\sum_{t_{i}<t} T\left(t-t_{i}\right) \gamma_{i} \triangleq I I_{1}(t)+I I_{2}(t) .
$$

Since $\left\{t_{i}^{j}\right\}, i \in Z, j=0, \pm 1, \pm 2, \ldots$, are equipotentially almost periodic, then by Lemma 35 in [20], for any $\epsilon>0$, there exist relatively dense sets of real numbers $\Omega_{2}$ and integers $\Omega_{3}$ such that the following relations hold:

(1) For every $\tau \in \Omega_{2}$, there exists at least one number $q \in \Omega_{3}$ such that

$$
\left|t_{i}^{q}-\tau\right|<\epsilon, \quad i \in Z
$$


(2) $\left\|\beta_{i+q}-\beta_{i}\right\|<\epsilon, q \in \Omega_{3}, i \in Z$.

So,

$$
\begin{aligned}
\left\|I_{1}(t+\tau)-I I_{1}(t)\right\| & =\left\|\sum_{t_{i}<t+\tau} T\left(t+\tau-t_{i}\right) \beta_{i}-\sum_{t_{i}<t} T\left(t-t_{i}\right) \beta_{i}\right\| \\
& \leq \sum_{t_{i}<t}\left\|T\left(t-t_{i}\right)\right\|\left\|\beta_{i+q}-\beta_{i}\right\| \\
& \leq \sum_{t_{i}<t} M e^{-\delta\left(t-t_{i}\right)} \epsilon<\frac{M}{1-e^{-\delta \gamma}} \epsilon .
\end{aligned}
$$

This shows $I I_{1} \in A P_{T}(R, X)$. It remains to show $I I_{2} \in P A P_{T}^{0}(R, X)$. For any $r>0$, there exist $i(r), j(r)$ such that

$$
t_{i(r)-1}<-r \leq t_{i(r)}<\cdots<t_{j(r)} \leq r<t_{j(r)+1} .
$$

Since $\gamma_{i} \in \operatorname{PAP}_{0}(Z, X), M_{\gamma_{i}}=\sup _{i \in Z}\left\|\gamma_{i}\right\|<\infty$, so

$$
\begin{aligned}
\frac{1}{2 r} \int_{-r}^{r}\left\|I I_{2}(t)\right\| d t= & \frac{1}{2 r} \int_{-r}^{r}\left\|\sum_{t_{i}<t} T\left(t-t_{i}\right) \gamma_{i}\right\| d t \\
\leq & \frac{1}{2 r} \int_{-r}^{r} \sum_{t_{i}<t} M e^{-\delta\left(t-t_{i}\right)}\left\|\gamma_{i}\right\| d t \\
\leq & \frac{1}{2 r} \sum_{t_{i}<-r} M e^{-\delta\left(-r-t_{i}\right)}\left\|\gamma_{i}\right\| \int_{-r}^{r} e^{-\delta(t+r)} d t \\
& +\frac{1}{2 r} \sum_{-r<t_{i}<r}\left\|\gamma_{i}\right\| \int_{-r}^{r} M e^{-\delta\left(t-t_{i}\right)} d t \\
\leq & \frac{1}{2 r} \sum_{t_{i}<-r} \frac{M}{\delta} M_{\gamma_{i}} e^{-\delta\left(-r-t_{i}\right)}+\frac{1}{2 r} \sum_{-r<t_{i}<r} \frac{M}{\delta}\left\|\gamma_{i}\right\| \\
\leq & \frac{1}{2 r} \frac{M M_{\gamma_{i}}}{\delta} \frac{1}{1-e^{-\delta \gamma}}+\frac{M}{\delta} \frac{1}{t_{j(r)}-t_{i(r)}} \sum_{k=i(r)}^{j(r)}\left\|\gamma_{k}\right\| \\
\leq & \frac{1}{2 r} \frac{M M_{\gamma_{i}}}{\delta} \frac{1}{1-e^{-\delta \gamma}}+\frac{M}{\delta \gamma} \frac{1}{j(r)-i(r)} \sum_{k=i(r)}^{j(r)}\left\|\gamma_{k}\right\| .
\end{aligned}
$$

Since $\gamma_{i} \in P A P_{0}(Z, X)$, for $r \rightarrow \infty, j(r)-i(r) \rightarrow \infty$,

$$
\frac{1}{j(r)-i(r)} \sum_{k=i(r)}^{j(r)}\left\|\gamma_{k}\right\| \rightarrow 0, \quad \text { as } r \rightarrow \infty .
$$

Clearly, $\lim _{r \rightarrow \infty} \frac{1}{2 r} \frac{M M_{\gamma_{i}}}{\delta\left(1-e^{-\delta \gamma}\right)}=0$, so

$$
\frac{1}{2 r} \int_{-r}^{r}\left\|I I_{2}(t)\right\| d t \rightarrow 0, \quad \text { as } r \rightarrow \infty .
$$

Thus, $\sum_{t_{i}<} T\left(\cdot-t_{i}\right) I_{i}\left(\phi\left(t_{i}\right)\right) \in \operatorname{PAP}_{T}(R, X)$, then for every $\phi \in D, \Gamma \phi \in P A P_{T}(R, X)$. 
Step 2. For every $\phi \in D,\|\Gamma \phi\| \leq L_{0}$.

For every $\phi \in D$, by (A1) and (A3), we have

$$
\begin{aligned}
\|\Gamma \phi(t)\| & =\left\|\int_{-\infty}^{t} T(t-s) f(s, \phi(s)) d s+\sum_{t_{i}<t} T\left(t-t_{i}\right) I_{i}\left(\phi\left(t_{i}\right)\right)\right\| \\
& \leq \int_{-\infty}^{t} M e^{-\delta(t-s)}\|f(s, \phi(s))\| d s+\sum_{t_{i}<t} M e^{-\delta\left(t-t_{i}\right)}\left\|I_{i}\left(\phi\left(t_{i}\right)\right)\right\| \\
& \leq C_{1 L_{0}} \int_{-\infty}^{t} M e^{-\delta(t-s)} d s+C_{2 L_{0}} \sum_{t_{i}<t} M e^{-\delta\left(t-t_{i}\right)} \\
& \leq \frac{M}{\delta} C_{1 L_{0}}+\frac{M}{1-e^{-\delta \gamma}} C_{2 L_{0}} \leq L_{0},
\end{aligned}
$$

then $\|\Gamma \phi\| \leq L_{0}$.

Step 3. For every $\phi \in D, \Gamma \phi \in U P C(R, X)$.

Suppose $\phi \in D, t^{\prime}, t^{\prime \prime} \in\left(t_{i}, t_{i+1}\right), i \in Z, \epsilon>0$, since $\{T(t): t \geq 0\}$ is a $C_{0}$-semigroup (see $[35,36])$ and $\|T(t)\| \leq M e^{-\delta t}, t \geq 0$, there exists $0<\mu<\frac{\epsilon}{3 M C_{1 L_{0}}}$ such that $0<t^{\prime}-t^{\prime \prime}<\mu$ implies

$$
\left\|T\left(t^{\prime}-t^{\prime \prime}\right)-I\right\|<\min \left\{\frac{\delta \epsilon}{3 M C_{1 L_{0}}}, \frac{\left(1-e^{-\delta \gamma}\right) \epsilon}{3 M C_{2 L_{0}}}\right\}
$$

Then

$$
\begin{aligned}
\| \Gamma \phi & \left(t^{\prime}\right)-\Gamma \phi\left(t^{\prime \prime}\right) \| \\
= & \| \int_{-\infty}^{t^{\prime}} T\left(t^{\prime}-s\right) f(s, \phi(s)) d s-\int_{-\infty}^{t^{\prime \prime}} T\left(t^{\prime \prime}-s\right) f(s, \phi(s)) d s \\
& +\sum_{t_{i}<t^{\prime}} T\left(t^{\prime}-t_{i}\right) I_{i}\left(\phi\left(t_{i}\right)\right)-\sum_{t_{i}<t^{\prime \prime}} T\left(t^{\prime \prime}-t_{i}\right) I_{i}\left(\phi\left(t_{i}\right)\right) \| \\
\leq & \left\|\int_{-\infty}^{t^{\prime \prime}}\left[T\left(t^{\prime}-s\right)-T\left(t^{\prime \prime}-s\right)\right] f(s, \phi(s)) d s\right\|+\left\|\int_{t^{\prime \prime}}^{t^{\prime}} T\left(t^{\prime}-s\right) f(s, \phi(s)) d s\right\| \\
& +\left\|\sum_{t_{i}<t^{\prime \prime}}\left[T\left(t^{\prime}-t_{i}\right)-T\left(t^{\prime \prime}-t_{i}\right)\right] I_{i}\left(\phi\left(t_{i}\right)\right)\right\| \\
\leq & \int_{-\infty}^{t^{\prime \prime}}\left\|T\left(t^{\prime \prime}-s\right)\right\|\left\|T\left(t^{\prime}-t^{\prime \prime}\right)-I\right\|\|f(s, \phi(s))\| d s+\int_{t^{\prime \prime}}^{t^{\prime}}\left\|T\left(t^{\prime}-s\right)\right\|\|f(s, \phi(s))\| d s \\
& +\sum_{t_{i}<t^{\prime \prime}}\left\|T\left(t^{\prime \prime}-t_{i}\right)\right\|\left\|T\left(t^{\prime}-t^{\prime \prime}\right)-I\right\|\left\|I_{i}\left(\phi\left(t_{i}\right)\right)\right\| \\
\leq & C_{1 L_{0}} \frac{\delta \epsilon}{3 M C_{1 L_{0}}} \int_{-\infty}^{t^{\prime \prime}} M e^{-\delta\left(t^{\prime \prime}-s\right)} d s+C_{1 L_{0}} M \frac{\epsilon}{3 M C_{1 L_{0}}}+C_{2 L_{0}} \frac{\left(1-e^{-\delta \gamma}\right) \epsilon}{3 M C_{2 L_{0}}} \sum_{t_{i}<t^{\prime \prime}} M e^{-\delta\left(t^{\prime \prime}-t_{i}\right)} \\
< & \frac{\epsilon}{3}+\frac{\epsilon}{3}+\frac{\epsilon}{3}=\epsilon,
\end{aligned}
$$

which shows $\Gamma \phi \in U P C(R, X)$.

Combing Step 1, Step 2, and Step 3, it follows that $\Gamma D \subseteq D$. 
Step 4. $\Gamma$ is continuous.

Let $\left\{\phi_{n}\right\} \subset D \subset P C(R, X), \phi_{n} \rightarrow \phi$ in $P C(R, X)$ as $n \rightarrow \infty$. Then we can find a bounded subset $K \subseteq \Omega$ such that $R(\phi) \subseteq K, R\left(\phi_{n}\right) \subseteq K, n \in N$. By (A2), given $\epsilon>0$, there exists $\delta^{\prime}>0$ such that $x, y \in K$ and $\|x-y\|<\delta^{\prime}$ implies that

$$
\|f(t, x)-f(t, y)\|<\epsilon, \quad \text { for all } t \in R,
$$

and

$$
\left\|I_{i}(x)-I_{i}(y)\right\|<\epsilon, \quad \text { for all } i \in Z \text {. }
$$

For the above $\delta^{\prime}$, there exists $n_{0}$ such that $\left\|\phi_{n}(t)-\phi(t)\right\|<\delta^{\prime}$ for $n>n_{0}$ and $t \in R$, then for $n>n_{0}$, we have

$$
\left\|f\left(t, \phi_{n}(t)\right)-f(t, \phi(t))\right\|<\epsilon, \quad \text { for all } t \in R,
$$

and

$$
\left\|I_{i}\left(\phi_{n}\left(t_{i}\right)\right)-I_{i}\left(\phi\left(t_{i}\right)\right)\right\|<\epsilon, \quad \text { for all } i \in Z \text {. }
$$

Hence,

$$
\begin{aligned}
\| & \Gamma \phi_{n}(t)-\Gamma \phi(t) \| \\
& =\left\|\int_{-\infty}^{t} T(t-s)\left[f\left(s, \phi_{n}(s)\right)-f(s, \phi(s))\right] d s+\sum_{t_{i}<t} T\left(t-t_{i}\right)\left[I_{i}\left(\phi_{n}\left(t_{i}\right)\right)-I_{i}\left(\phi\left(t_{i}\right)\right)\right]\right\| \\
& \leq \int_{-\infty}^{t} M e^{-\delta(t-s)}\left\|f\left(s, \phi_{n}(s)\right)-f(s, \phi(s))\right\| d s+\sum_{t_{i}<t} M e^{-\delta\left(t-t_{i}\right)}\left\|I_{i}\left(\phi_{n}\left(t_{i}\right)\right)-I_{i}\left(\phi\left(t_{i}\right)\right)\right\| \\
& \leq \int_{-\infty}^{t} M e^{-\delta(t-s)} \epsilon d s+\sum_{t_{i}<t} M e^{-\delta\left(t-t_{i}\right)} \epsilon \\
& \leq\left[\frac{M}{\delta}+\frac{M}{1-e^{-\delta \gamma}}\right] \epsilon,
\end{aligned}
$$

from which it follows that $\Gamma$ is continuous.

Step 5. $D(t)=\{\Gamma \phi(t): \phi \in D\}$ is a relatively compact subset of $X$ for each $t \in R$.

For each $t \in R, 0<\epsilon<1, \phi \in D$, define

$$
\begin{aligned}
\Gamma^{\epsilon} \phi(t) & =\int_{-\infty}^{t-\epsilon} T(t-s) f(s, \phi(s)) d s+\sum_{t_{i}<t-\epsilon} T\left(t-t_{i}\right) I_{i}\left(\phi\left(t_{i}\right)\right) \\
& =T(\epsilon)\left[\int_{-\infty}^{t-\epsilon} T(t-\epsilon-s) f(s, \phi(s)) d s+\sum_{t_{i}<t-\epsilon} T\left(t-\epsilon-t_{i}\right) I_{i}\left(\phi\left(t_{i}\right)\right)\right] \\
& =T(\epsilon) \Gamma \phi(t-\epsilon) .
\end{aligned}
$$

Since $\{\Gamma \phi(t-\epsilon): \phi \in D\}$ is bounded and $T(\epsilon)$ is compact, $\left\{\Gamma^{\epsilon} \phi(t): \phi \in D\right\}$ is a relatively compact subset of $X$. Moreover, for $\epsilon$ is small enough, the point $t$ and $t-\epsilon$ belong to the 
same interval of continuity of $\phi$, then

$$
\Gamma \phi(t)-\Gamma^{\epsilon} \phi(t)=\int_{t-\epsilon}^{t} T(t-s) f(s, \phi(s)) d s
$$

So,

$$
\left\|\Gamma \phi(t)-\Gamma^{\epsilon} \phi(t)\right\| \leq \int_{t-\epsilon}^{t} M e^{-\delta(t-s)}\|f(s, \phi(s))\| d s \leq M C_{1 L_{0}} \epsilon
$$

Thus, $D(t)=\{\Gamma \phi(t): \phi \in D\}$ is a relatively compact subset of $X$ for each $t \in R$.

By Step 3, we know that $\{\Gamma \phi: \phi \in D\}$ is equipotentially continuous at each interval $\left(t_{i}, t_{i+1}\right)(i \in Z)$. Since $\{\Gamma \phi: \phi \in D\} \subset(P C)_{h}^{0}(R, X)$ and $\{\Gamma \phi: \phi \in D\}$ satisfies the conditions of Lemma 4.2, $\{\Gamma \phi: \phi \in D\}$ is a relatively compact set, then $\Gamma$ is a compact operator. Since $D$ is a closed convex set, it follows from the Schauder fixed point theorem that $\Gamma$ has a fixed point $\phi$ in $D$. So, the fixed point $\phi$ satisfies the integral equation

$$
\phi(t)=\int_{-\infty}^{t} T(t-s) f(s, \phi(s)) d s+\sum_{t_{i}<t} T\left(t-t_{i}\right) I_{i}\left(\phi\left(t_{i}\right)\right)
$$

for all $t \in R$. Fix $a, a \neq t_{i}, i \in Z$, we have

$$
\phi(a)=\int_{-\infty}^{a} T(a-s) f(s, \phi(s)) d s+\sum_{t_{i}<a} T\left(a-t_{i}\right) I_{i}\left(\phi\left(t_{i}\right)\right) .
$$

Since $\{T(t): t \geq 0\}$ is a $C_{0}$-semigroup, so

$$
\begin{aligned}
\phi(t) & \\
= & \int_{-\infty}^{t} T(t-s) f(s, \phi(s)) d s+\sum_{t_{i}<t} T\left(t-t_{i}\right) I_{i}\left(\phi\left(t_{i}\right)\right) \\
= & \int_{-\infty}^{a} T(t-s) f(s, \phi(s)) d s+\sum_{t_{i}<a} T\left(t-t_{i}\right) I_{i}\left(\phi\left(t_{i}\right)\right) \\
& +\int_{a}^{t} T(t-s) f(s, \phi(s)) d s+\sum_{a<t_{i}<t} T\left(t-t_{i}\right) I_{i}\left(\phi\left(t_{i}\right)\right) \\
= & T(t-a) \phi(a)+\int_{a}^{t} T(t-s) f(s, \phi(s)) d s+\sum_{a<t_{i}<t} T\left(t-t_{i}\right) I_{i}\left(\phi\left(t_{i}\right)\right) .
\end{aligned}
$$

Hence, $\phi$ is a piecewise pseudo almost periodic mild solution to Eq. (1.1).

The following existence result is based on the contraction principle.

Theorem 4.5 Assume the following conditions hold:

$\left(\mathrm{A1}^{\prime}\right) A$ is the infinitesimal generator of an exponentially stable $C_{0}$-semigroup $\{T(t): t \geq 0\}$; that is, there exist numbers $\delta, M>0$ such that $\|T(t)\| \leq M e^{-\delta t}, t \geq 0$. 
(A2') $f \in \operatorname{PAP}_{T}(R \times \Omega, X)$, and $f$ satisfies the Lipschitz condition in the following sense:

$$
\|f(t, x)-f(t, y)\| \leq L_{1}\|x-y\|, \quad t \in R, x, y \in \Omega,
$$

where $L_{1}>0$.

(A3') $I_{i}$ is a pseudo almost periodic sequence, and there exists a number $L_{2}>0$ such that

$$
\left\|I_{i}(x)-I_{i}(y)\right\| \leq L_{2}\|x-y\|
$$

for all $x, y \in \Omega, i \in Z$.

If $\frac{M}{\delta} L_{1}+\frac{M}{1-e^{-\delta \gamma}} L_{2}<1$, then Eq. (1.1) has a piecewise pseudo almost periodic solution.

Proof Define the operator $\Gamma$ on $\operatorname{PAP}_{T}(R, X) \cap U P C(R, X)$ as in the proof of Theorem 4.4. Fix $\phi \in P A P_{T}(R, X) \cap U P C(R, X)$, by $\left(\mathrm{A}^{\prime}\right)$ and $\left(\mathrm{A}^{\prime}\right)$, we have

$$
\|f(t, \phi(t))\| \leq\|f(t, \phi(t))-f(t, 0)\|+\|f(t, 0)\| \leq L_{1}\|\phi(t)\|+\|f(t, 0)\|,
$$

and

$$
\left\|I_{i}\left(\phi\left(t_{i}\right)\right)\right\| \leq\left\|I_{i}\left(\phi\left(t_{i}\right)\right)-I_{i}(0)\right\|+\left\|I_{i}(0)\right\| \leq L_{2}\left\|\phi\left(t_{i}\right)\right\|+\left\|I_{i}(0)\right\| .
$$

Then $f(\cdot, \phi(\cdot)) \in P C(R, X)$ and $I_{i}\left(\phi\left(t_{i}\right)\right)$ is a bounded sequence, it follows from the proof of Theorem 4.4 that $\Gamma \phi \in P A P_{T}(R, X) \cap U P C(R, X)$. So, $\Gamma\left(P A P_{T}(R, X) \cap U P C(R, X)\right) \subset$ $P A P_{T}(R, X) \cap U P C(R, X)$. It suffices now to show that the operator $\Gamma$ has a fixed point in $\operatorname{PAP}_{T}(R, X) \cap U P C(R, X)$. For $\phi, \varphi \in \operatorname{PAP}_{T}(R, X) \cap U P C(R, X)$,

$$
\begin{aligned}
\| \Gamma & \phi(t)-\Gamma \varphi(t) \| \\
& =\left\|\int_{-\infty}^{t} T(t-s)[f(s, \phi(s))-f(s, \varphi(s))] d s+\sum_{t_{i}<t} T\left(t-t_{i}\right)\left[I_{i}\left(\phi\left(t_{i}\right)\right)-I_{i}\left(\varphi\left(t_{i}\right)\right)\right]\right\| \\
& \leq \int_{-\infty}^{t} M e^{-\delta(t-s)}\|f(s, \phi(s))-f(s, \varphi(s))\| d s+\sum_{t_{i}<t} M e^{-\delta\left(t-t_{i}\right)}\left\|I_{i}\left(\phi\left(t_{i}\right)\right)-I_{i}\left(\varphi\left(t_{i}\right)\right)\right\| \\
& \leq \int_{-\infty}^{t} M e^{-\delta(t-s)} L_{1}\|\phi(s)-\varphi(s)\| d s+\sum_{t_{i}<t} M e^{-\delta\left(t-t_{i}\right)} L_{2}\left\|\phi\left(t_{i}\right)-\varphi\left(t_{i}\right)\right\| \\
& \leq\left[\int_{-\infty}^{t} L_{1} M e^{-\delta(t-s)} d s+\sum_{t_{i}<t} L_{2} M e^{-\delta\left(t-t_{i}\right)}\right]\|\phi-\varphi\| \\
& \leq\left[\frac{M}{\delta} L_{1}+\frac{M}{1-e^{-\delta \gamma}} L_{2}\right]\|\phi-\varphi\| .
\end{aligned}
$$

Since $\frac{M}{\delta} L_{1}+\frac{M}{1-e^{-\delta \gamma}} L_{2}<1, \Gamma$ is a contraction, $\Gamma$ has a fixed point in $P A P_{T}(R, X) \cap U P C(R, X)$, then Eq. (1.1) has a piecewise pseudo almost periodic solution.

Finally, we investigate the stability of a piecewise pseudo almost periodic solution to Eq. (1.1). By using the generalized Gronwall-Bellman inequality (see Lemma 1 in [20]) and Lipschitz conditions, it can be formulated as follows. 
Theorem 4.6 Suppose the conditions of Theorem 4.5 hold. Assume further that $\frac{\ln \left(1+M L_{2}\right)}{\gamma}+$ $M L_{1}-\delta<0$, then Eq. (1.1) has an exponentially stable piecewise pseudo almost periodic solution.

Proof By Theorem 4.5, we know that Eq. (1.1) has a mild piecewise pseudo almost periodic solution $u(t)$, by using the integral form of Eq. (1.1), if $t>\sigma, \sigma \neq t_{i}, i \in Z$,

$$
u(t)=T(t-\sigma) u(\sigma)+\int_{\sigma}^{t} T(t-s) f(s, u(s)) d s+\sum_{\sigma<t_{i}<t} T\left(t-t_{i}\right) I_{i}\left(u\left(t_{i}\right)\right)
$$

Let $u(t)=u(t, \sigma, \varphi)$ and $v(t)=v(t, \sigma, \psi)$ be two solutions of Eq. (1.1), then

$$
\begin{aligned}
& u(t)=T(t-\sigma) \varphi+\int_{\sigma}^{t} T(t-s) f(s, u(s)) d s+\sum_{\sigma<t_{i}<t} T\left(t-t_{i}\right) I_{i}\left(u\left(t_{i}\right)\right), \\
& v(t)=T(t-\sigma) \psi+\int_{\sigma}^{t} T(t-s) f(s, v(s)) d s+\sum_{\sigma<t_{i}<t} T\left(t-t_{i}\right) I_{i}\left(v\left(t_{i}\right)\right) .
\end{aligned}
$$

So,

$$
\begin{aligned}
\|u(t)-v(t)\| \leq & \|T(t-\sigma)[\varphi-\psi]\|+\left\|\int_{\sigma}^{t} T(t-s)[f(s, u(s))-f(s, v(s))] d s\right\| \\
& +\left\|\sum_{\sigma<t_{i}<t} T\left(t-t_{i}\right)\left[I_{i}\left(u\left(t_{i}\right)\right)-I_{i}\left(v\left(t_{i}\right)\right)\right]\right\| \\
\leq & \|T(t-\sigma)\|\|\varphi-\psi\|+\int_{\sigma}^{t}\|T(t-s)\|\|f(s, u(s))-f(s, v(s))\| d s \\
& +\sum_{\sigma<t_{i}<t}\left\|T\left(t-t_{i}\right)\right\|\left\|I_{i}\left(u\left(t_{i}\right)\right)-I_{i}\left(v\left(t_{i}\right)\right)\right\| \\
\leq & M e^{-\delta(t-\sigma)}\|\varphi-\psi\|+\int_{\sigma}^{t} M L_{1} e^{-\delta(t-s)}\|u(s)-v(s)\| d s \\
& +\sum_{\sigma<t_{i}<t} M L_{2} e^{-\delta\left(t-t_{i}\right)}\left\|u\left(t_{i}\right)-v\left(t_{i}\right)\right\| .
\end{aligned}
$$

Then

$$
\begin{aligned}
e^{\delta t}\|u(t)-v(t)\| \leq & M e^{\delta \sigma}\|\varphi-\psi\|+\int_{\sigma}^{t} M L_{1} e^{\delta s}\|u(s)-v(s)\| d s \\
& +\sum_{\sigma<t_{i}<t} M L_{2} e^{\delta t_{i}}\left\|u\left(t_{i}\right)-v\left(t_{i}\right)\right\| .
\end{aligned}
$$

Let $y(t)=e^{\delta t}\|u(t)-v(t)\|,(4.7)$ can be rewritten in the following form:

$$
y(t) \leq M y(\sigma)+\int_{\sigma}^{t} M L_{1} y(s) d s+\sum_{\sigma<t_{i}<t} M L_{2} y\left(t_{i}\right) .
$$

By the generalized Gronwall-Bellman inequality, we have

$$
y(t) \leq M y(\sigma) \prod_{\sigma<t_{i}<t}\left(1+M L_{2}\right) e^{\int_{\sigma}^{t} M L_{1} d s}=M y(\sigma) \prod_{\sigma<t_{i}<t}\left(1+M L_{2}\right) e^{M L_{1}(t-\sigma)} .
$$


Since $\gamma=\inf _{i \in Z}\left(t_{i+1}-t_{i}\right)>0$, we have

$$
y(t) \leq M y(\sigma)\left(1+M L_{2}\right)^{\frac{t-\sigma}{\gamma}} e^{M L_{1}(t-\sigma)}=M y(\sigma) e^{\left[\frac{\ln \left(1+M L_{2}\right)}{\gamma}+M L_{1}\right](t-\sigma)} .
$$

That is,

$$
\|u(t)-v(t)\| \leq M\|\varphi-\psi\| e^{\left[\frac{\ln \left(1+M L_{2}\right)}{\gamma}+M L_{1}-\delta\right](t-\sigma)} .
$$

Because $\frac{\ln \left(1+M L_{2}\right)}{\gamma}+M L_{1}-\delta<0$, then Eq. (1.1) has an exponentially stable piecewise pseudo almost periodic solution. This completes the proof.

Example 4.7 Consider the following problem:

$$
\left\{\begin{array}{l}
\frac{\partial}{\partial t} u(t, \xi)=\frac{\partial^{2}}{\partial \xi^{2}} u(t, \xi)+\frac{1}{15}(\sin t+\sin \sqrt{2} t+g(t)) \sin u(t, \xi) \\
\quad t \in R, t \neq t_{i}, i \in Z, \xi \in[0, \pi] \\
\triangle u\left(t_{i}, \xi\right)=\beta_{i} u\left(t_{i}, \xi\right), \quad i \in Z, \xi \in[0, \pi] \\
u(t, 0)=u(t, \pi)=0, \quad t \in R
\end{array}\right.
$$

where $g \in U P C(R, R)$ satisfies $|g(t)| \leq 1(t \in R)$ and $\lim _{r \rightarrow \infty} \frac{1}{2 r} \int_{r}^{r}|g(t)| d t=0, \beta_{i}=\frac{1}{15}(\sin i+$ $\sin \sqrt{2} i+g(i))$ and $t_{i}=i+\frac{1}{4}|\sin i-\sin \sqrt{2} i|$.

Define $X=L^{2}[0, \pi]$, let $A u=u^{\prime \prime}, u \in D(A)=H_{0}^{1}[0, \pi] \cap H^{2}[0, \pi]$. Clearly, $A$ is the infinitesimal generator of an analytic compact semigroup $\{T(t): t \geq 0\}$ on $X$ (refer to [21]) and $\|T(t)\| \leq e^{-t}(t \geq 0)$ with $M=\delta=1 .\left\{t_{i}^{j}\right\}, i \in Z, j=0, \pm 1, \pm 2, \ldots$, are equipotentially almost periodic (refer to p.198 in [20]) and

$$
\begin{aligned}
t_{i}^{1} & =t_{i+1}-t_{i}=1+\frac{1}{4}|\sin (i+1)-\sin \sqrt{2}(i+1)|-\frac{1}{4}|\sin i-\sin \sqrt{2} i| \\
& \geq 1-\frac{1}{4}|\sin (i+1)-\sin i-[\sin \sqrt{2}(i+1)-\sin \sqrt{2} i]| \\
& \geq 1-\frac{1}{2}\left|\sin \frac{1}{2} \cos \frac{2 i+1}{2}\right|-\frac{1}{2}\left|\sin \frac{\sqrt{2}}{2} \cos \frac{\sqrt{2}(2 i+1)}{2}\right| \\
& \geq 1-\frac{1}{2} \sin \frac{1}{2}-\frac{1}{2} \sin \frac{\sqrt{2}}{2}>\frac{2}{5} .
\end{aligned}
$$

So, $\gamma=\inf _{i \in Z}\left(t_{i+1}-t_{i}\right)>\frac{2}{5}>0$. Let $f(t, u)=\frac{1}{15}(\sin t+\sin \sqrt{2} t+g(t)) \sin u, I_{i}(u)=\beta_{i} u$. Clearly, both $f$ and $I_{i}$ satisfy the assumptions given in Theorem 4.5 and Theorem 4.6 with $L_{1}=\frac{1}{5}$, $L_{2}=\frac{1}{5}$, respectively. Moreover, $\frac{M}{\delta} L_{1}+\frac{M}{1-e^{-\delta \gamma}} L_{2}<\frac{1}{5}+\frac{1}{5\left(1-e^{-\frac{2}{5}}\right)}<1, \frac{\ln \left(1+M L_{2}\right)}{\gamma}+M L_{1}-\delta<\frac{5}{2} \ln \frac{6}{5}+$ $\frac{1}{5}-1<0$, all conditions in Theorem 4.6 are satisfied. Hence, Eq. (4.8) has an exponentially stable piecewise pseudo almost periodic solution.

The authors declare that they have no competing interests. 


\section{Acknowledgements}

This research was supported by the National Natural Science Foundation of China (No. 11071048). The authors are thankful the referees for their careful reading of the manuscript and insightful comments.

Received: 24 September 2012 Accepted: 21 December 2012 Published: 14 January 2013

\section{References}

1. Zhang, CY: Pseudo almost periodic solutions of some differential equations. J. Math. Anal. Appl. 181(1), 62-76 (1994)

2. Zhang, CY: Pseudo almost periodic solutions of some differential equations, II. J. Math. Anal. Appl. 192(2), 543-561 (1995)

3. Agarwal, RP, de Andrade, B, Cuevas, C: On type of periodicity and ergodicity to a class of fractional order differential equations. Adv. Differ. Equ. (2010). doi:10.1155/2010/179750

4. Agarwal, RP, Cuevas, C, Soto, H: Pseudo-almost periodic solutions of a class of semilinear fractional differential equations. J. Appl. Math. Comput. 37, 625-634 (2011)

5. Agarwal, RP, Diagana, T, Hernandez, E: Weighted pseudo almost periodic solutions to some partial neutral functional differential equations. J. Nonlinear Convex Anal. 8(3), 397-415 (2007)

6. Amir, B, Maniar, L: Composition of pseudo-almost periodic functions and Cauchy problems with operator of nondense domain. Ann. Math. Blaise Pascal 6(1), 1-11 (1999)

7. Cuevas, C, Hernandez, E: Pseudo-almost periodic solutions for abstract partial functional differential equation. Appl. Math. Lett. 22, 534-538 (2009)

8. Cuevas, C, Pinto, M: Existence and uniqueness of pseudo almost periodic solutions of semilinear Cauchy problems with non dense domain. Nonlinear Anal. TMA 45(1), 73-83 (2001)

9. Diagana, T: Pseudo almost periodic solutions to some differential equations. Nonlinear Anal. TMA 60(7), 1277-1286 (2005)

10. Diagana, T: Weighted pseudo almost periodic functions and applications. C. R. Math. Acad. Sci. Paris 343(10), 643-646 (2006)

11. Diagana, T: Stepanov-like pseudo almost periodic functions and their applications to differential equations. Commun. Math. Anal. 3(1), 9-18 (2007)

12. Diagana, T: Stepanov-like pseudo-almost periodicity and its applications to some nonautonomous differential equations. Nonlinear Anal. TMA 69(12), 4277-4285 (2008)

13. Diagana, T, Herandez, E, Rabello, M: Pseudo almost periodic solutions to some non-autonomous neutral functional differential equations with unbounded delay. Math. Comput. Model. 45, 1241-1252 (2007)

14. Ding, HS, Liang, J, N'Guerekata, GM, Xiao, TJ: Pseudo-almost periodicity of some nonautonomous evolution equations with delay. Nonlinear Anal. TMA 67, 1412-1418 (2007)

15. Li, HX, Huang, FL, Li, JY: Composition of pseudo almost-periodic functions and semilinear differential equations. J. Math. Anal. Appl. 255(2), 436-446 (2001)

16. Li, HX, Zhang, LL: Stepanov-like pseudo almost periodicity and semilinear differential equations with uniform continuity. Results Math. 59, 43-61 (2011)

17. Li, YK, Wang, C: Pseudo almost periodic functions and pseudo almost periodic solutions to dynamic equations on time scales. Adv. Differ. Equ. (2012). doi:10.1186/1687-1847-2012-77

18. Long, W, Ding, HS: Composition theorems of Stepanov almost periodic functions and Stepanov-like pseudo-almost periodic functions. Adv. Differ. Equ. (2011). doi:10.1155/2011/654695

19. Rogovchenko, YV: Nonlinear impulse evolution systems and applications to population models. J. Math. Anal. Appl. 207, 300-315 (1997)

20. Samoilenko, AM, Perestyuk, NA: Impulsive Differential Equations. World Scientific, Singapore (1995)

21. Wang, JR, Xiang, X, Peng, Y: Periodic solutions of semilinear impulsive periodic system on Banach space. Nonlinear Anal. TMA 71, e1344-e1353 (2009)

22. Cuevas, C, N'Guerekata, G, Rabelo, M: Mild solutions for impulsive neutral functional differential equations with state-dependent delay. Semigroup Forum 80, 375-390 (2010)

23. Hernandez, E, Henriquez, HR, McKibben, M: Existence results for abstract impulsive second order neutral functional differential equations. Nonlinear Anal. TMA 70(7), 2736-2751 (2009)

24. Hernandez, E, Rabello, M, Henriquez, HR: Existence of solutions for impulsive partial neutral functional equations. J. Math. Anal. Appl. 331, 1135-1158 (2007)

25. Hernandez, E, Pierri, M, Goncalves, G: Existence results for an impulsive abstract partial differential equation with state-dependent delay. Comput. Math. Appl. 52, 411-420 (2006)

26. Henriquez, HR, de Andrade, B, Rabelo, M: Existence of almost periodic solutions for a class of abstract impulsive differential equations. ISRN Math. Anal. (2011). doi:10.5402/2011/632687

27. Liu, JW, Zhang, CY: Existence and stability of almost periodic solutions for impulsive differential equations. Adv. Differ. Equ. (2012). doi:10.1186/1687-1847-2012-34

28. Stamov, GT, Alzabut, JO: Almost periodic solutions for abstract impulsive differential equations. Nonlinear Anal. TMA 72(5), 2457-2464 (2010)

29. Liang, J, Zhang, J, Xiao, TJ: Composition of pseudo almost automorphic and asymptotically almost automorphic function. J. Math. Anal. Appl. 340, 1493-1499 (2008)

30. Agarwal, RP, de Andrade, B, Cuevas, C: Weighted pseudo almost periodic solutions of a class of semilinear fractional differential equations. Nonlinear Anal., Real World Appl. 11, 3532-3554 (2010)

31. Zhang, CY: Almost Periodic Type Functions and Ergodicity. Science Press, Beijing (2003)

32. Cuevas, C, Hernandez, E, Rabelo, M: The existence of solutions for impulsive neutral functional differential equations. Comput. Math. Appl. 58(4), 744-757 (2009)

33. Henriquez, $\mathrm{H}$, Lizama, $\mathrm{C}$ : Compact almost automorphic solutions to integral equations with infinite delay. Nonlinear Anal. TMA 71, 6029-6037 (2009)

34. Agarwal, RP, Cuevas, C, Soto, H, El-Gebeily, M: Asymptotic periodicity for some evolution equations in Banach spaces. Nonlinear Anal. TMA 74, 1769-1798 (2011) 
35. Pazy, A: Semigroups of Linear Operators and Applications to Partial Differential Equations. Springer, New York (1983)

36. Agarwal, RP, Cuevas, C, dos Santos, JP: Analytic resolvent operator and existence results for fractional integro-differential equations. J. Abstr. Differ. Equ. Appl. 2(2), 26-47 (2012)

doi:10.1186/1687-1847-2013-11

Cite this article as: Liu and Zhang: Composition of piecewise pseudo almost periodic functions and applications to abstract impulsive differential equations. Advances in Difference Equations 2013 2013:11.

Submit your manuscript to a SpringerOpen ${ }^{\circ}$ journal and benefit from:

- Convenient online submission

Rigorous peer review

- Immediate publication on acceptance

- Open access: articles freely available online

- High visibility within the field

- Retaining the copyright to your article

Submit your next manuscript at $>$ springeropen.com 